Short communication

\title{
Actin-independent trafficking of cochlear connexin 26 to non-lipid raft gap junction plaques
}

\author{
Jean Defourny*, Nicolas Thelen, Marc Thiry \\ GIGA-Neurosciences, Unit of Cell and Tissue Biology, University of Liège, C.H.U. B36, B-4000, Liège, Belgium
}

\section{A R T I C L E I N F O}

\section{Article history:}

Received 29 May 2018

Received in revised form

13 December 2018

Accepted 24 January 2019

Available online $\mathrm{xxx}$

\section{Keywords:}

Cochlea

GJB2

Connexin 26

Gap junctions

Actin

Lipid rafts

\begin{abstract}
A B S T R A C T
Hereditary hearing loss affects about 1 per 1000 children. Mutations in GJB2, which encodes the connexin 26 protein (Cx26) involved in cochlear homeostasis, are found in about $50 \%$ of patients with autosomal recessive non-syndromic hearing loss. Deciphering the trafficking pathway of cochlear Cx26 in situ should represent an advance in understanding the pathogenic significance of many of these mutations. Connexins trafficking and delivery to lipid raft-associated gap junction plaques usually requires successively microtubule and actin networks. Here we show that cochlear Cx26 exhibits an unusual trafficking pathway. We observed that Cx26 assembly occurs in non-lipid raft membrane domains and that junctional plaques are devoid of actin and associated zonula occludens proteins. Using cytoskeleton-disrupting drugs in organotypic culture, we found that cochlear Cx26 gap junction assembly requires microtubules but not actin filaments. Altogether, our data provide an unexpected insight into Cx26 trafficking pathway and gap junction assembly in the cochlea.
\end{abstract}

() 2019 Elsevier B.V. All rights reserved.

\section{Introduction}

Hearing loss is the most common congenital sensory deficit. About $1-3$ in 1000 children are affected at birth or during early childhood by severe hearing loss, which is defined as prelingual deafness, with at least half of all cases attributable to genetic causes (Korver et al., 2017). Despite extraordinary genetic heterogeneity, mutations in one gene, GJB2, which encodes the connexin 26 protein (Cx26) and is involved in inner ear homeostasis, are found in up to $50 \%$ of patients with autosomal recessive non-syndromic hearing loss (Kelsell et al., 1997). Beside this non-syndromic form of deafness, GJB2 mutations cause several types of syndromic hearing loss associated with skin diseases with variable prognosis (Avshalumova et al., 2014; Lilly et al., 2016; Xu and Nicholson, 2013). In mammals, sounds are perceived through mechanosensory hair cells located within the sensory epithelium of the cochlea (i.e. the organ of Corti). Within the organ of Corti, sensory inner and outer hair cells and non-sensory supporting cells are organized in a regular mosaic pattern that extends along the basalto-apical axis of the cochlear duct. Cx26 gap junction protein, which assembles to form channels between cochlear supporting cells,

\footnotetext{
* Corresponding author.

E-mail address: jean.defourny@uliege.be (J. Defourny).
}

allows the rapid removal of $\mathrm{K}^{+}$away from the base of sensory hair cells, resulting in the recycling of this ion back to the endolymph to maintain cochlear homeostasis (Kikuchi et al., 2000). However, gap junctions may serve additional roles in the cochlea, such as providing networks for nutrient transfer (Chang et al., 2008; Jagger and Forge, 2015). Cx26 and Cx30 (encoded by the deafness gene GJB6 (Grifa et al., 1999)) are the two most abundantly expressed gap junction proteins in the cochlea and form heteromeric and heterotypic channels between adjacent supporting cells, from the spiral limbus to the cochlear spiral ligament (Ahmad et al., 2003; Sun et al., 2005) (Fig. 1A). Although the two channel components Cx26 and $\mathrm{Cx} 30$ are well characterized, the gap junction plaque (GJP) assembly mechanisms occurring in situ remain largely unknown. Of note, however, is the fact that these two proteins are not functionally equivalent, since $\mathrm{Cx} 26$ has been shown as the key organizer of the gap junction macromolecular complex (Kamiya et al., 2014). Beside mutations that affect the Cx26 channel function itself, many of the disease-causing mutations in GJB2 impair the trafficking and delivery of Cx26 to the cell surface, what prevents the formation of gap junctions (Ambrosi et al., 2013; Hoang Dinh et al., 2009; Xu and Nicholson, 2013). Thus, deciphering the trafficking pathway of cochlear Cx26 should represent an advance in understanding the pathogenic significance of these mutations.

Gap junction assembly usually occurs in a "two-step mechanism" which requires successively microtubules and actin 


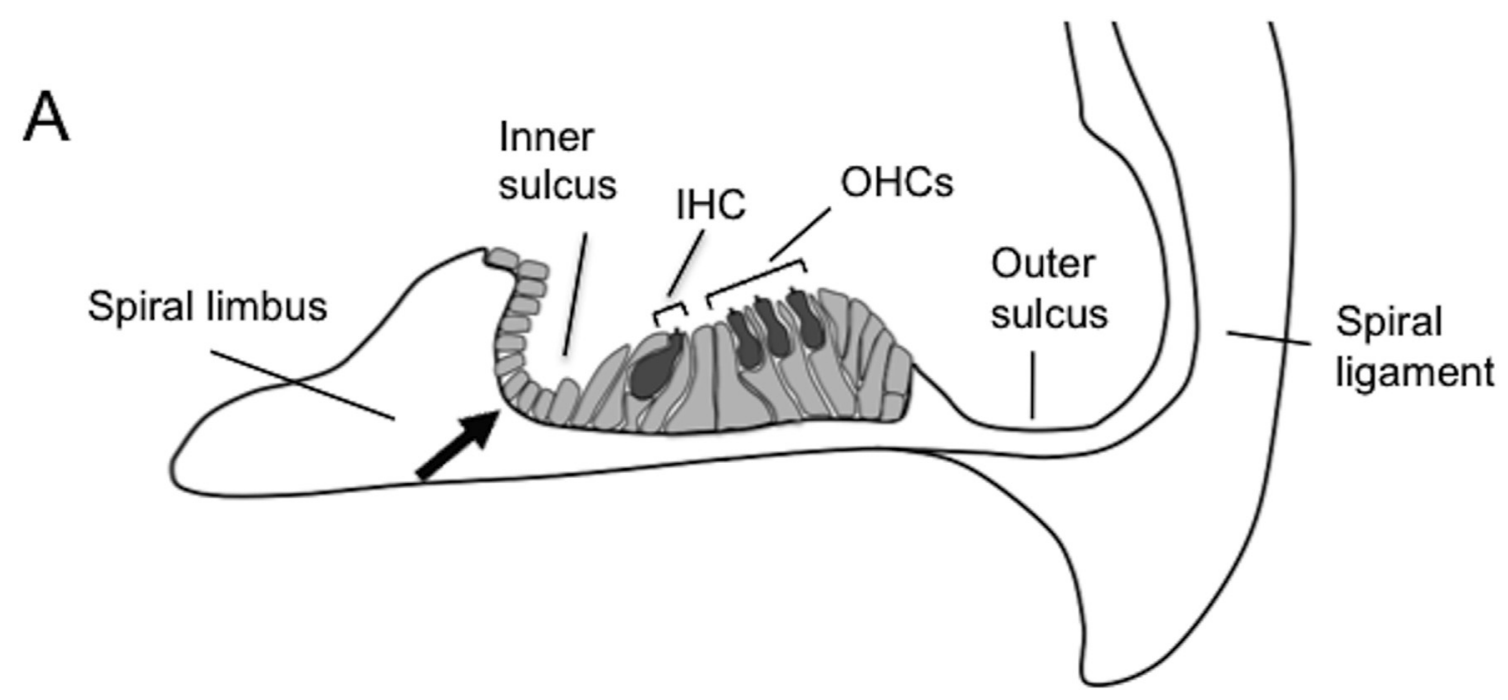

B

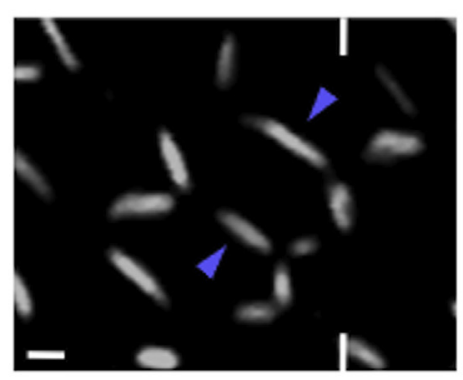

Cx26

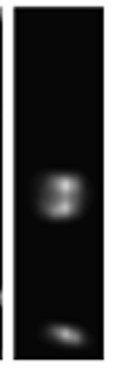

F-actin
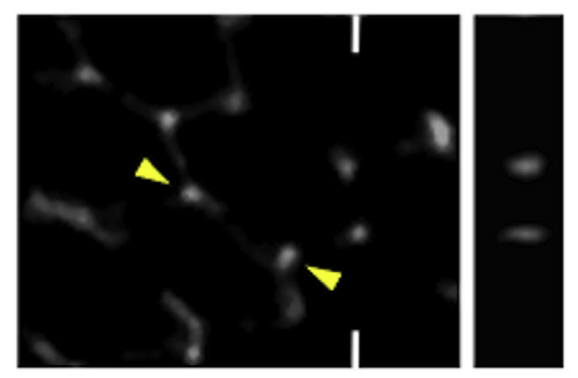

\section{Cx26 / F-actin}

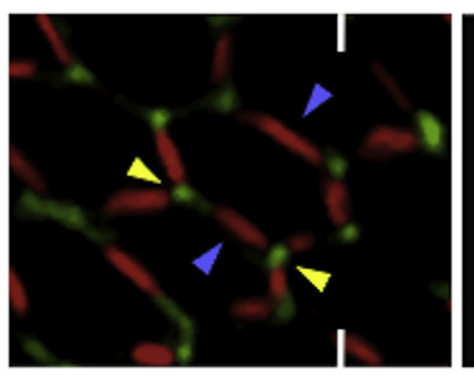

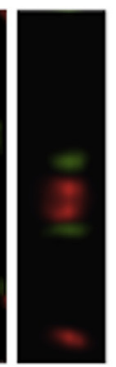

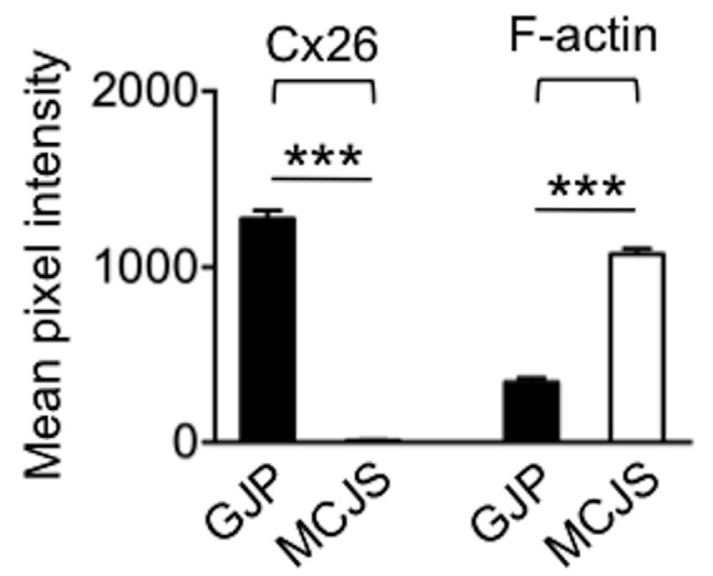

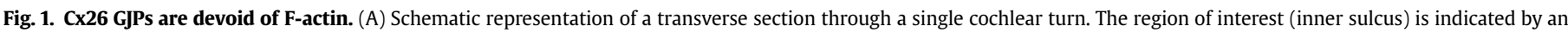

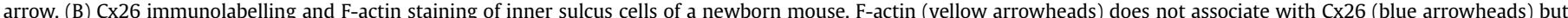

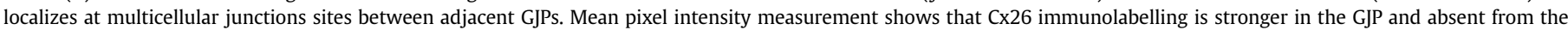

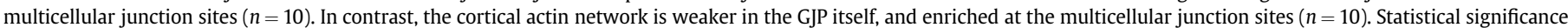

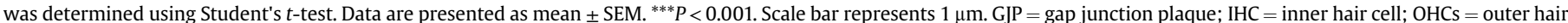
cells; $\mathrm{MCJS}=$ multicellular junction site. (For interpretation of the references to colour in this figure legend, the reader is referred to the Web version of this article.)

cytoskeletal components. First, hexameric connexons assembled in the trans-Golgi network are trafficked along microtubules to the non-junctional plasma membrane (Koval et al., 1997; Lauf et al., 2002; Musil and Goodenough, 1993). Secondly, hemichannels associate with cortical actin through actin-binding proteins zonula occludens (ZOs) which regulate delivery of connexins from the periphery to the GJP (Hervé et al., 2014; Thévenin et al., 2013). The membrane lipid environment is critical for gap junction assembly and function (Cascio, 2005; Defamie and Mesnil, 2012). Junctional connexins assemble into lipid raft microdomains whereas nonjunctional connexins are present in non-lipid raft fractions (Defamie and Mesnil, 2012; Hunter et al., 2005; Musil and 
Goodenough, 1991). Because of the relatively short half-life of connexins (usually $1-5 \mathrm{~h}$ ), the junctional plaque is in a dynamic state, constantly remodeled through both recruitment of newly synthesized connexons to the periphery and endocytosis of older components from the center of the plaque (Gaietta et al., 2002).

Here we show that cochlear Cx26 exhibits an unexpected trafficking pathway towards non-lipid raft gap junction plaques. We observed that junctional $\mathrm{C} \times 26$ are devoid of F-actin and associated ZO-1 and ZO-2. Using cytoskeleton-disrupting drugs in organotypic culture, we found that the assembly of Cx26 into GJPs requires microtubules but not actin networks. Therefore, unlike most connexins, the trafficking of cochlear Cx26 towards non-lipid raft GJPs occurs in a kind of "one-step mechanism", only microtubuledependent.

\section{Materials and methods}

\subsection{Animals}

Mice of the BALB/c strain were group-housed in the animal facility of the University of Liège under standard conditions with food and water ad libitum and were maintained on a 12-h light/dark cycle. All animals were taken care in accordance with the Declaration of Helsinki and following the guidelines of the Belgian ministry of agriculture in agreement with EC laboratory animal care and use regulation (2010/63/UE, 22 September 2010).

\subsection{Tissue processing and immunostainings}

Cochleae of newborn mice were fixed for $2 \mathrm{~h}$ in $4 \%$ formaldehyde. Whole-mount cochleae or organotypic explants were incubated overnight at $4{ }^{\circ} \mathrm{C}$ with primary antibodies directed against connexin 26 (rabbit polyclonal antibody; 1:500; Invitrogen), ZO-1 (rat monoclonal antibody, 1:50; Santa Cruz Biotechnology) and ZO-2 (mouse monoclonal antibody, 1:50; Santa Cruz
Biotechnology). TRITC-conjugated phalloidin (1:500; SigmaAldrich) was used as an F-actin marker. Lipid rafts were labelled using FITC-conjugated Cholera Toxin B subunit $(1 \mu \mathrm{g} / \mathrm{mL}$; SigmaAldrich). Tissues were then incubated for $1 \mathrm{~h}$ with either Rhodamine Red X- or FITC-conjugated goat anti-mouse, anti-rabbit or anti-rat IgGs secondary antibodies (Jackson Immunoresearch Laboratories).

\subsection{In vitro organotypic assay}

Organs of Corti were isolated from newborn mice and cultured onto Millicell Culture Insert (Millipore) as previously described (Defourny et al., 2015). Organotypic cultures were incubated for $2 \mathrm{~h}$ with dimethyl sulfoxide (DMSO, vehicle), nocodazole $(100 \mu \mathrm{M}$; Sigma-Aldrich) or cytochalasin D (10 $\mu \mathrm{M}$; Sigma-Aldrich).

\subsection{Confocal microscopy, image analysis and quantification}

Confocal fluorescence images were acquired using the Olympus Fluoview FV1000 confocal system (Olympus Europa GmbH). For comparison between different culture conditions, all preparations were analysed at the same time, using the same acquisition parameters. For each culture condition, 150 inner sulcus cells and 150 GJPs were quantified and measured from three independent experiments and data were plotted. Inner sulcus cells were randomly chosen and GJPs were measured using Image software. Mean pixel intensities in GJPs and in adjacent multicellular junction sites were measured using ImageJ software.

\subsection{Statistics}

All data are presented as mean \pm SEM. Data were statistically analysed using two-tailed Student's $t$-test or one-way ANOVA followed by Dunnett's post-test. $P$-values $<0.05$ were considered significant $\left({ }^{* * *} P<0.001\right)$.

\section{Cx26}

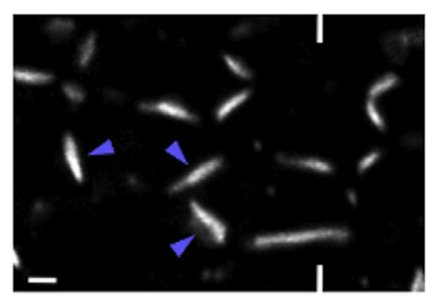

Cx26 / Cholera Toxin B

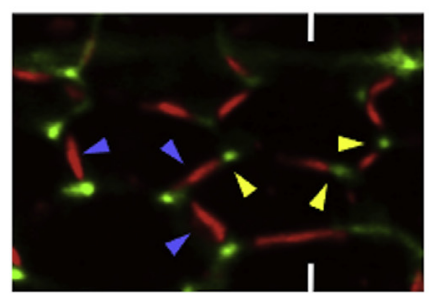

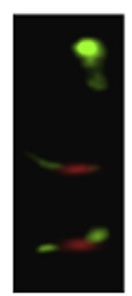

\section{Cholera Toxin B}
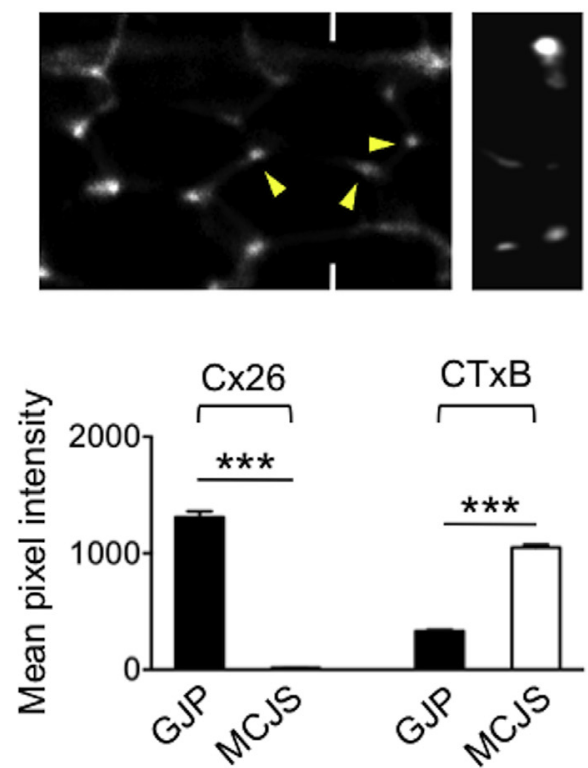

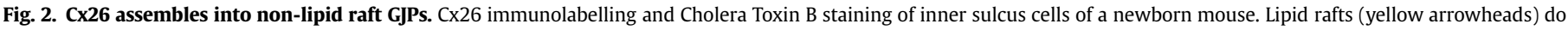

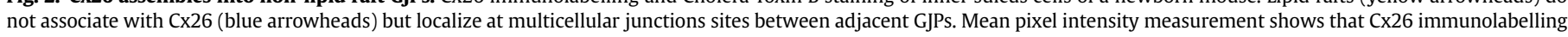

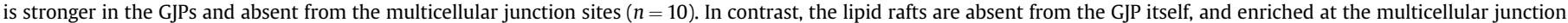

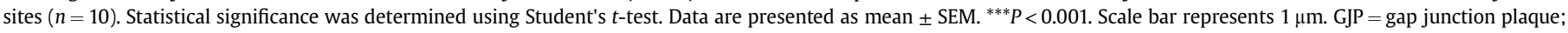
$\mathrm{MCJS}=$ multicellular junction site. (For interpretation of the references to colour in this figure legend, the reader is referred to the Web version of this article.) 


\subsection{Data availability}

The data that support the findings of this study are available from the corresponding author upon reasonable request.

\section{Results and discussion}

\subsection{Cx26 gap junctions are devoid of cortical actin and associated ZOS}

Once they have reached the plasma membrane, most connexins are linked to the submembrane actin network through ZOs. ZOs contain three PDZ domains and connexins interact with them via a PDZ-binding motif (Hervé et al., 2014; Thévenin et al., 2013). Interestingly, the genomic duplication and overexpression of TJP2/ ZO-2 causes a progressive non-syndromic hearing loss in humans (Walsh et al., 2010). We then examined whether cochlear Cx26 gap junctions associate with cortical actin and $\mathrm{ZO}$ proteins in inner sulcus cells of newborn mice. These non-sensory cells, located close to the inner hair cell layer, were previously considered to address the role of Cx26 in the assembly of the gap junction macromolecular complex in the cochlea (Kamiya et al., 2014) (the inner sulcus is indicated by an arrow in Fig. 1A). Surprisingly, we found that F-actin does not associate with Cx26 but rather localizes at multicellular junctions sites between adjacent GJPs (Fig. 1B). Similar distributions were observed in outer sulcus cells, which are non-sensory cells located between the outer hair cell rows and the spiral ligament (Fig. S1A). F-actin and Cx26 therefore display mutually exclusive localizations. Moreover, a co-immunolabelling of Cx26 and ZO- 1 or ZO-2 revealed that none of these two proteins associate with junctional Cx26 (Fig. S2). Although rather surprising, these findings are consistent with the fact that murine and human Cx26 do not contain any predicted PDZ-binding motif at their Cterminal extremity (Thévenin et al., 2013; Sheng and Sala, 2001).
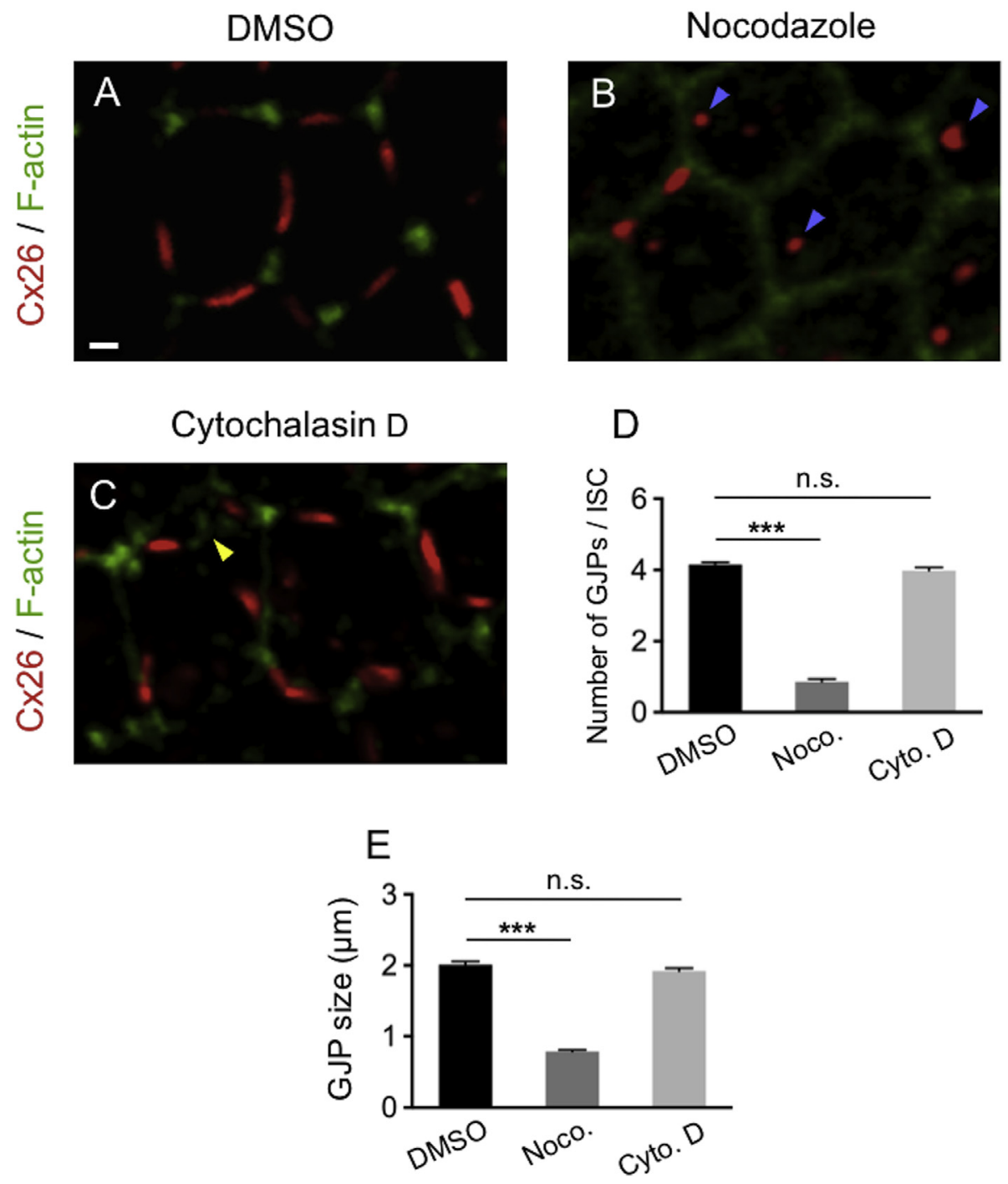

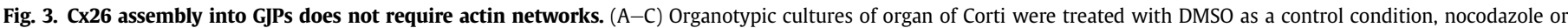

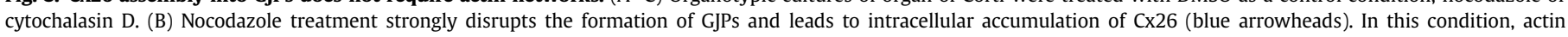

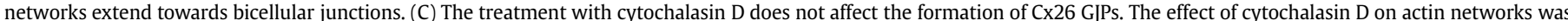

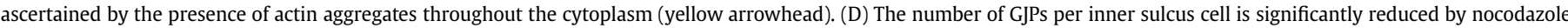

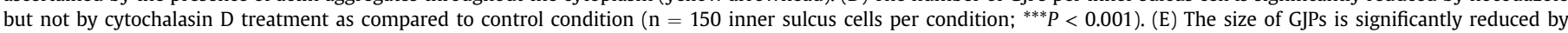

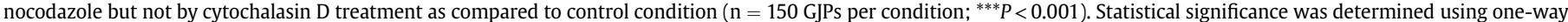

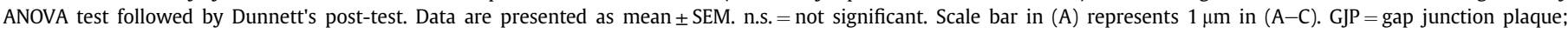
ISC = inner sulcus cell. (For interpretation of the references to colour in this figure legend, the reader is referred to the Web version of this article.) 
The cytoplasmic tail is the most divergent domain with varying sizes among the connexins and is believed to interact with a variety of molecules (Kotini and Mayor, 2015). Since Cx26 is one of the smallest connexins with a short C-terminal tail, this could explain why Cx26 cannot associate with F-actin and/or actin-binding proteins.

\subsection{Cx26 assembles into non-lipid raft gap junction plaques}

The composition of the plasma membrane lipid bilayer has a strong effect on ion channels (Cascio, 2005; Defamie and Mesnil, 2012). In vitro studies have shown that Cx26 has a low affinity for cholesterol and a preference for tight association with anionic phospholipids (Hung and Yarovsky, 2011; Locke and Harris, 2009). Lipid rafts, which are known to modulate gap junction communication (Defamie and Mesnil, 2012), contain a high quantity of cholesterol and a low amount of anionic phospholipids (Pike, 2003). Using Cholera Toxin B subunit as a relevant marker of these membrane microdomains (Janes et al., 1999), we tested whether Cx26 assembles into lipid rafts or not in inner sulcus cells. We found that Cx26 does not associate with lipid rafts, which are exclusively present at multicellular junction sites between adjacent GJPs (Fig. 2). Similar mutually exclusive localization patterns are observed in outer sulcus cells (Fig. S1B). These findings are consistent with previous data showing that Cx26 is excluded from lipid rafts in cell culture (Locke et al., 2005; Schubert et al., 2002). Moreover, we found that F-actin specifically associates with lipid rafts at multicellular junction sites, as expected (Chichili and Rodgers, 2009) (Fig. S3). Therefore, these data reveal an unusual submembrane compartmentalization for cochlear Cx26.

\subsection{Actin-independent assembly of Cx26 into gap junction plaques}

Distinct cytoskeletal tracks are required for trafficking lipid raftassociated or non-lipid raft-associated proteins to the plasma membrane (Jacob et al., 2003). Lipid raft-associated proteins travel to the cell periphery along microtubules and use actin filaments in the cell cortex to reach lipid raft membrane microdomains. In contrast, non-lipid raft-associated proteins traffic along microtubules and reach their plama membrane destination without requiring actin tracks (Jacob et al., 2003). We then tested whether microtubules and/or actin networks are needed for delivery of Cx26 to non-lipid raft GJPs in inner sulcus cells. To this end, explants of organ of Corti isolated from newborn mice were treated with nocodazole and cytochalasin D as microtubules- and actindisrupting drugs, respectively. Nocodazole treatment disturbed the transport of Cx26 to the cell periphery and strongly reduced the formation of GJPs in inner sulcus cells (Fig. 3B,D,E). In this condition, actin networks extend towards bicellular junctions, likely because microtubule depolymerization promotes reassembly of actin stress fibers (Ezratty et al., 2005). In agreement with previous in vitro data (George et al., 1999; Martin et al., 2001), an intact microtubule network is thus required for trafficking cochlear Cx26 from the interior of the cell to the plasma membrane. In contrast, we found that cytochalasin D did not significantly affect the assembly of Cx26 into GJPs (Fig. 3C-E). The effect of cytochalasin D on actin networks was ascertained by the presence of actin aggregates throughout the cytoplasm (Schliwa, 1982) (arrowhead on Fig. 3C). These findings suggest that, unlike other connexins, Cx26 uses an actin-independent trafficking pathway to assemble into non-lipid raft GJPs in the cochlea. This is consistent with recent data showing that $\mathrm{C} \times 30$ does not rely on actin network for GJP assembly when expressed as heteromeric channels with Cx26 in inner sulcus cells (Defourny et al., 2019). In contrast, in Deiters' cells (i.e. the supporting cells which surround the outer hair cells), Cx30 homomeric channels associate with the actin network, which likely promotes the recruitement of $\mathrm{Cx} 30$ from the peripheral region to the GJP (Defourny et al., 2019). Together, these results suggest that intrinsic Cx26 gap junction assembly features dominate the ones of Cx30 in situ.

Whereas most connexins assemble in a "two-step mechanism", i.e. in a microtubule- and actin-dependent fashion, our data suggest that cochlear Cx26 most likely traffics in a kind of "one-step process", only microtubule-dependent (Fig. 4). Such «actin bypass » pathway could be needed to ensure the relatively fast turnover rate of Cx26 (Traub et al., 1987). This could be the reason why Cx26 oligomers are already found in the endoplasmic reticulum (Diez et al., 1999) and can reach the cell surface via a route bypassing the Golgi complex (George et al., 1999; Martin et al., 2001). Although connexins usually reach the plasma membrane by traveling along the secretory pathway (Laird, 2006), this nonclassical route used by $\mathrm{Cx} 26$ should be a faster process than trafficking through the Golgi (Grieve and Rabouille, 2011). In this context, an in vitro cell-free transcription/translation system has shown that Cx26 exhibits a singular membrane-integration behaviour and integrates directly in a post-translational manner into plasma membranes. Protein-cleavage studies of Cx26 integrated into plasma membranes indicated a similar native transmembrane topography to that of Cx26 integrated co-translationally
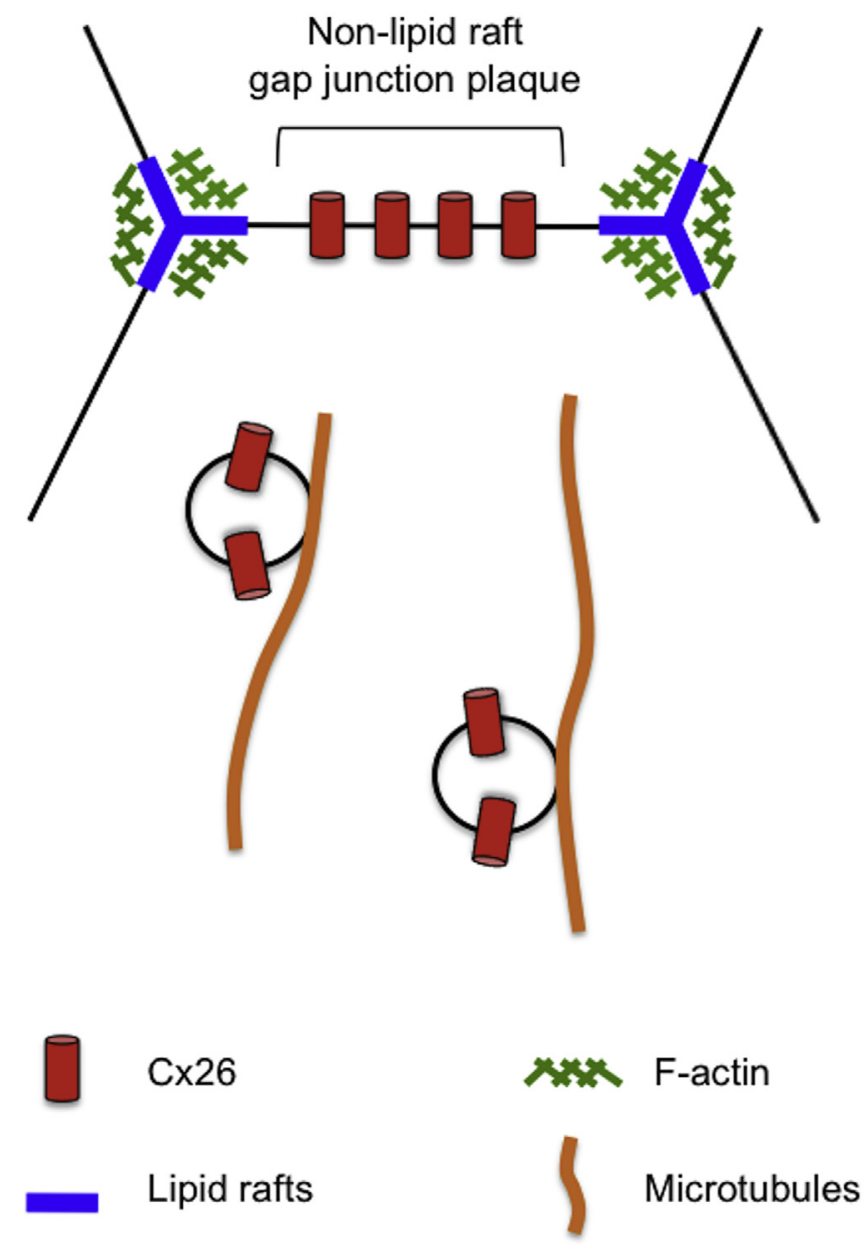

Fig. 4. A model for how Cx26 traffics and assembles into GJPs in the cochlea. Cx26 travels along microtubules and assembles into non-lipid raft GJPs in an actinindependent fashion. F-actin associates with lipid rafts at multicellular junction sites between adjacent GJPs. 
into microsomes (Ahmad and Evans, 2002; Zhang et al., 1996). Cx26 oligomerization and assembly into hemichannels thus occurs independently of the conventional biogenesis of gap junctions involving connexin trafficking and oligomerization via membrane components of the secretory pathway (Ahmad and Evans, 2002).

Cx26 channel activity has been shown to be highly sensitive to cholesterol concentration (Locke and Harris, 2009). As a consequence, it should be of interest to determine how junctional Cx26 are specifically retained in non-lipid raft compartments. In a mouse embryo fibroblast cell line, the distibution of $\mathrm{Cx} 43$ at cell interfaces has been shown to be controlled by the transmembrane protein ephrin-B1 (Davy et al., 2006). Interestingly, a protein from the same family, ephrin-B2, was found to be specifically expressed in cochlear supporting cells (Defourny et al., 2015). Since gap junction communication is promoted at ephrin/ephrin interfaces within a cell compartment (Davy et al., 2006), it is tempting to speculate that ephrin-B2 might regulate Cx26 distribution and gap junction communication between adjacent supporting cells.

\section{Conclusion}

Altogether, our data provide an unexpected insight into Cx26 trafficking pathway and gap junction assembly in the cochlea. Since many of the disease-causing mutations in GJB2 impair the trafficking and delivery of Cx26 to the cell surface (Ambrosi et al., 2013; Hoang Dinh et al., 2009; Xu and Nicholson, 2013), our findings should help further research aimed to decipher the pathogenic significance of these mutations.

\section{Competing financial interests}

The authors declare no competing financial interests.

\section{Acknowledgements}

We thank the GIGA-Cell Imaging platform. This work was supported by the Belgian Fonds de la Recherche Scientifique - FNRS.

\section{Appendix A. Supplementary data}

Supplementary data to this article can be found online at https://doi.org/10.1016/j.heares.2019.01.020.

\section{References}

Ahmad, S., Chen, S., Sun, J., Lin, X., 2003. Connexins 26 and 30 are co-assembled to form gap junctions in the cochlea of mice. Biochem. Biophys. Res. Commun. 307, 362-368. https://doi.org/10.1016/S0006-291X(03)01166-5.

Ahmad, S., Evans, W.H., 2002. Post-translational integration and oligomerization of connexin 26 in plasma membranes and evidence of formation of membrane pores : implications for the assembly of gap junctions. Biochem. J. 365, 603-699. https://doi.org/10.1042/bj20011572.

Ambrosi, C., Walker, A.E., Depriest, A.D., Cone, A.C., Lu, C., Badger, J., Skerrett, I.M., Sosinsky, G.E., 2013. Analysis of trafficking, stability and function of human connexin 26 gap junction channels with deafness-causing mutations in the fourth transmembrane helix. PLoS One 8, e70916. https://doi.org/10.1371/ journal.pone.0070916.

Avshalumova, L., Fabrikant, J., Koriakos, A., 2014. Overview of skin diseases linked to connexin gene mutations. Int. J. Dermatol. 53, 192-205. https://doi.org/10.1111/ ijd.12062.

Cascio, M., 2005. Connexins and their environment : effects of lipids composition on ion channels. Biochim. Biophys. Acta 1711, 142-153. https://doi.org/10.1016/ j.bbamem.2004.12.001.

Chang, Q., Tang, W., Ahmad, S., Zhou, B., Lin, X., 2008. Gap junction mediated intercellular metabolite transfer in the cochlea is compromised in connexin 30 null mice. PLoS One 3, e4088. https://doi.org/10.1371/journal.pone.0004088.

Chichili, G.R., Rodgers, W., 2009. Cytoskeleton-membrane interactions in membrane raft structure. Cell. Mol. Life Sci. 66, 2319-2328. https://doi.org/10.1007/ s00018-009-0022-6.

Davy, A., Bush, J.O., Soriano, P., 2006. Inhibition of gap junction communication at ectopic Eph/ephrin boundaries underlies craniofrontonasal syndrome. PLoS
Biol. 4, e315. https://doi.org/10.1371/journal.pbio.0040315.

Defamie, N., Mesnil, M., 2012. The modulation of gap-junctional intercellular communication by lipid rafts. Biochim. Biophys. Acta 1818, 1866-1869. https:/ doi.org/10.1016/j.bbamem.2011.09.023.

Defourny, J., Mateo Sánchez, S., Schoonaert, L., Robberecht, W., Davy, A., Nguyen, L. Malgrange, B., 2015. Cochlear supporting cell transdifferentiation and integration into hair cell layers by inhibition of ephrin-B2 signalling. Nat. Commun. 6 , 7017. https://doi.org/10.1038/ncomms8017.

Defourny, J., Thelen, N., Thiry, M., 2019. Cochlear connexin 30 homomeric and heteromeric channels exhibit distinct assembly mechanisms. Mech. Dev. 155 8-14. https://doi.org/10.1016/j.mod.2018.10.001.

Diez, J.A., Ahmad, S., Evans, W.H., 1999. Assembly of heteromeric connexons in guinea-pig liver en route to the Golgi apparatus, plasma membrane and gap junctions. Eur. J. Biochem. 262, 142-148. https://doi.org/10.1046/j.1432-1327. 1999.00343.x.

Ezratty, E.J., Partridge, M.A., Gundersen, G.G., 2005. Microtubule-induced focal adhesion disassembly is mediated by dynamin and focal adhesion kinase. Nat. Cell Biol. 7, 581-590. https://doi.org/10.1038/ncb1262.

Gaietta, G., Deerinck, T.J., Adams, S.R., Bouwer, J., Tour, O., Laird, D.W., Sosinsky, G.E. Tsien, R.Y., Ellisman, M.H., 2002. Multicolor and electron microscopic imaging of connexin trafficking. Science 296, 503-507. https://doi.org/10.1126/science. 1068793.

George, C.H., Kendall, J.M., Evans, W.H., 1999. Intracellular trafficking pathways in the assembly of connexins into gap junctions. J. Biol. Chem. 274, 8678-8685. https://doi.org/10.1074/jbc.274.13.8678.

Grieve, A.G., Rabouille, C., 2011. Golgi bypass : skirting around the heart of classical secretion. Cold Spring Harb. Perspect. Biol. 3, a005298. https://doi.org/10.1101/ cshperspect.a005298.

Grifa, A., Wagner, C.A., D'Ambrosio, L., Melchionda, S., Bernardi, F., Lopez-Bigas, N. Rabionet, R., Arbones, M., Monica, M.D., Estivill, X., et al., 1999. Mutations in GJB6 cause nonsyndromic autosomal dominant deafness at DFNA3 locus. Nat. Genet. 23, 16-18. https://doi.org/10.1038/12612.

Hervé, J.C., Derangeon, M., Sarrouilhe, D., Bourmeyster, N., 2014. Influence of the scaffolding protein Zonula Occludens (ZOs) on membrane channels. Biochim. Biophys. Acta 1838, 595-604. https://doi.org/10.1016/j.bbamem.2013.07.006.

Hoang Dinh, E., Ahmad, S., Chang, Q., Tang, W., Stong, B., Lin, X., 2009. Diverse deafness mechanisms of connexin mutations revealed by studies using in vitro approaches and mouse models. Brain Res. 1277, 52-69. https://doi.org/10.1016/ j.brainres.2009.02.008.

Hung, A., Yarovsky, I., 2011. Gap junction hemichannel interactions with zwitterionic lipid, anionic lipid, and cholesterol : molecular simulation studies. Biochemistry 50, 1492-1504. https://doi.org/10.1021/bi1004156.

Hunter, A.W., Barker, R.J., Zhu, C., Gourdie, R.G., 2005. Zonula occludens-1 alters connexin43 gap junction size and organization by influencing channel accretion. Mol. Biol. Cell 16, 5686-5698. https://doi.org/10.1091/mbc.E05-08-0737.

Jacob, R., Heine, M., Alfalah, M., Naim, H.Y., 2003. Distinct cytoskeletal tracks direct individual vesicle populations to the apical membrane of epithelial cells. Curr. Biol. 13, 607-612. https://doi.org/10.1016/S0960-9822(03)00188-X.

Jagger, D.J., Forge, A., 2015. Connexins and gap junctions in the inner ear-it's not just about $\mathrm{K}+$ recycling. Cell Tissue Res. 360, 633-644. https://doi.org/10.1007/ s00441-014-2029-z.

Janes, P.W., Ley, S.C., Magee, A.I., 1999. Aggregation of lipid rafts accompagnies signaling via the T cell antigen receptor. J. Cell Biol. 147, 447-461. https://doi. org/10.1083/jcb.147.2.447.

Kamiya, K., Yum, S.W., Kurebayashi, N., Muraki, M., Ogawa, K., Karasawa, K. Miwa, A., Guo, X., Gotoh, S., Sugitani, Y., et al., 2014. Assembly of the cochlear gap junction macromolecular complex requires connexin 26. J. Clin. Invest. 124 1598-1607. https://doi.org/10.1172/JCI67621.

Kelsell, D.P., Dunlop, J., Stevens, H.P., Lench, N.J., Liang, J.N., Parry, G., Mueller, R.F. Leigh, I.M., 1997. Connexin 26 mutations in hereditary non-syndromic sensorineural deafness. Nature 387, 80-83. https://doi.org/10.1038/387080a0.

Kikuchi, T., Kimura, R.S., Paul, D.L., Takasaka, T., Adams, J.C., 2000. Gap junction systems in the mammalian cochlea. Brain Res. Brain Res. Rev. 32, 163-166. https://doi.org/10.1016/S0165-0173(99)00076-4.

Kotini, M., Mayor, R., 2015. Connexins in migration during development and cancer. Dev. Biol. 401, 143-151. https://doi.org/10.1016/j.ydbio.2014.12.023.

Korver, A.M., Smith, R.J., Van Camp, G., Schleiss, M.R., Bitner-Glindzicz, M.A. Lustig, L.R., Usami, S.I., Boudewyns, A.N., 2017. Congenital hearing loss. Nat. Rev. Dis. Primers 3, 16094. https://doi.org/10.1038/nrdp.2016.94.

Koval, M., Harley, J.E., Hick, E., Steinberg, H.E., 1997. Connexin46 is retained as monomers in a trans-Golgi compartment of osteoblastic cells. J. Cell Biol. 137, 847-857. https://doi.org/10.1083/jcb.137.4.847.

Laird, D.W., 2006. Life cycle of connexins in health and disease. Biochem. J. 394 527-543. https://doi.org/10.1042/BJ20051922.

Lauf, U., Giepmans, B.N., Lopez, P., Braconnot, S., Chen, S.C., Falk, M.M., 2002. Dynamic trafficking and delivery of connexons to the plasma membrane and accretion to gap junctions in living cells. Proc. Natl. Acad. Sci. U.S.A. 99, 10446-10451. https://doi.org/10.1073/pnas.162055899.

Lilly, E., Sellitto, C., Milstone, L.M., White, T.W., 2016. Connexin channels in congenital skin disorders. Semin. Cell Dev. Biol. 50, 4-12. https://doi.org/10. 1016/j.semcdb.2015.11.018.

Locke, D., Harris, A.L., 2009. Connexin channels and phospholipids : association and modulation. BMC Biol. 7, 52. https://doi.org/10.1186/1741-7007-7-52.

Locke, D., Liu, J., Harris, A.L., 2005. Lipid rafts prepared by different methods contain different connexin channels, but gap junction are not lipid rafts. Biochemistry 
44, 13027-13042. https://doi.org/10.1021/bi050495a.

Martin, P.E., Blundell, G., Ahmad, S., Errington, R.J., Evans, W.H., 2001. Multiple pathways in the trafficking and assembly of connexin 26, 32 and 43 into gap junction intercellular communication channels. J. Cell Sci. 114, 3845-3855.

Musil, L.S., Goodenough, D.A., 1991. Biochemical analysis of connexin43 intracellular transport, phosphorylation, and assembly into gap junctional plaques. J. Cell Biol. 115, 1357-1374. https://doi.org/10.1083/jcb.115.5.1357.

Musil, L.S., Goodenough, D.A., 1993. Multisubunit assembly of an integral plasma membrane channel protein, gap junction connexin43, occurs after exit from the ER. Cell 74, 1065-1077. https://doi.org/10.1016/0092-8674(93)90728-9.

Pike, L.J., 2003. Lipid rafts : bringing order to chaos. J. Lipid Res. 44, 655-667. https://doi.org/10.1194/jlr.R200021-JLR200.

Schliwa, M., 1982. Action of cytochalasin D on cytoskeletal networks. J. Cell Biol. 92 79-91. https://doi.org/10.1083/jcb.92.1.79.

Schubert, A.L., Schubert, W., Spray, D.C., Lisanti, M.P., 2002. Connexin family members target to lipid raft domains and interact with caveolin-1. Biochemistry 41, 5754-5764. https://doi.org/10.1021/bi0121656.

Sheng, M., Sala, C., 2001. PDZ domains and the organization of supramolecular complexes. Annu. Rev. Neurosci. 24, 1-29. https://doi.org/10.1146/annurev. neuro.24.1.1.

Sun, J., Ahmad, S., Chen, S., Tang, W., Zhang, Y., Chen, P., Lin, X., 2005. Cochlear gap junctions coassembled from Cx26 and 30 show faster intercellular Ca2+ signaling than homomeric counterparts. Am. J. Physiol. Cell Physiol. 288, C613-C623. https://doi.org/10.1152/ajpcell.00341.2004.

Thévenin, A.F., Kowal, T.J., Fong, J.T., Kells, R.M., Fisher, C.G., Falk, M.M., 2013. Proteins and mechanisms regulating gap-junction assembly, internalization, and degradation. Physiology (Bethesda) 28, 93-116. https://doi.org/10.1152/physiol. 00038.2012 .

Traub, O., Look, J., Paul, D., Willecke, K., 1987. Cyclic adenosine monophosphate stimulates biosynthesis and phosphorylation of the $26 \mathrm{kDa}$ gap junction protein in cultured mouse hepatocytes. Eur. J. Cell Biol. 43, 48-54.

Walsh, T., Pierce, S.B., Lenz, D.R., Brownstein, Z., Dagan-Rosenfeld, O., Shahin, H., Roeb, W., McCarthy, S., Nord, A.S., Gordon, C.R., et al., 2010. Genomic duplication and overexpression of TJP2/ZO-2 leads to altered expression of apoptosis genes in progressive nonsyndromic hearing loss DFNA51. Am. J. Hum. Genet. 87, 101-109. https://doi.org/10.1016/j.ajhg.2010.05.011.

$\mathrm{Xu}$, J., Nicholson, B.J., 2013. The role of connexins in ear and skin physiology functional insights from disease-associated mutations. Biochim. Biophys. Acta 1828, 167-178. https://doi.org/10.1016/j.bbamem.2012.06.024.

Zhang, J.T., Chen, M., Foote, C.I., Nicholson, B.J., 1996. Membrane integration of in vitro-translated gap junctional proteins : co- and post-translational mechanisms. Mol. Biol. Cell 7, 471-482. 